Note

\section{Production of Molecular Hydrogen by a Continuous Culture under Laboratory Conditions}

\author{
Jihn Sang Kıм,* Kazutoshi Ito, \\ Kazuo IzaKi and Hajime TAKAHASHI \\ Department of Agricultural Chemistry, \\ Faculty of Agriculture, \\ Tohoku University, \\ Sendai 980, Japan
}

Received March 5, 1987

In a previous paper, we reported on the production of molecular hydrogen by a semi-contiruous outdoor culture of Rhodopseudomonas sphaeroides $\mathbf{B} 6,{ }^{1)}$ which was isolated in the Bangkok area, Thailand. ${ }^{2)}$ During the semicontinuous culture, a part of the culture medium was withdrawn and replaced by fresh medium from time to time. It is obvious that a true continuous culture, in which fresh medium is fed during the daytime, is preferable to a semi-continuous culture. However, the rate of feeding of the fresh medium is a problem in a continuous outdoor culture, because the rate of consumption of the electron donor, and therefore the rate of hydrogen production, varies depending on the strength of illumination and temperature of the cultivation. To improve the efficiency of hydrogen production, we examined the conditions for a continuous culture including selection of bacterial strains and the effect of the illumination interval.

Rhodopseudomonas sphaeroides B6 was used throughout this work. As reported previously, ${ }^{1)}$ this strain is more stable in a semi-continuous culture than $R$. sphaeroides B5, which was used for the outdoor batch culture. ${ }^{3)}$ As a basal medium, the modified Ormerod medium ${ }^{4)}$ was used, except that $p$-aminobenzoic acid was omitted. Two kinds of media were employed. Medium A was the basal medium with $25 \mathrm{~mm}$ DL-lactate and $5 \mathrm{~mm} \mathrm{~L}$-glutamate added. Medium B contained $47 \mathrm{~mm}$ DL-lactate and $10 \mathrm{~mm} \mathrm{L-}$ glutamate.

For the continuous culture under laboratory conditions, we used the apparatus for hydrogen production shown diagrammatically in Fig. 1. A glass bottle (light receiving area, $22 \times 14 \mathrm{~cm}$; width, $5 \mathrm{~cm}$ ), containing $1,000 \mathrm{ml}$ of culture medium, was connected to a medium reservoir and a receiver for the spent medium. Sampling of the medium was performed from the top of the bottle. The medium was stirred by a Teflon-coated magnetic bar $(2.7 \mathrm{~cm}$ in length and $0.9 \mathrm{~cm}$ in diameter, and at about $200 \mathrm{rpm}$ ). After inoculation, the surface of the culture medium was sealed with liquid paraffin. The bottle was incubated in a glass water bath at $33^{\circ} \mathrm{C}$ under illumination ( $c a .30 \mathrm{klux}$ ) by two $200 \mathrm{~W}$ incandescent bulbs. A wet-type flow meter (model WK-0.5; Shinagawa Co., Ltd., Tokyo) connected to the gas outlet of the bottle was used to measure the total and the rate of gas evolution. The evolved gas "was analyzed for hydrogen and carbon dioxide with a Yanagimoto G1800-T gas chromatograph as described

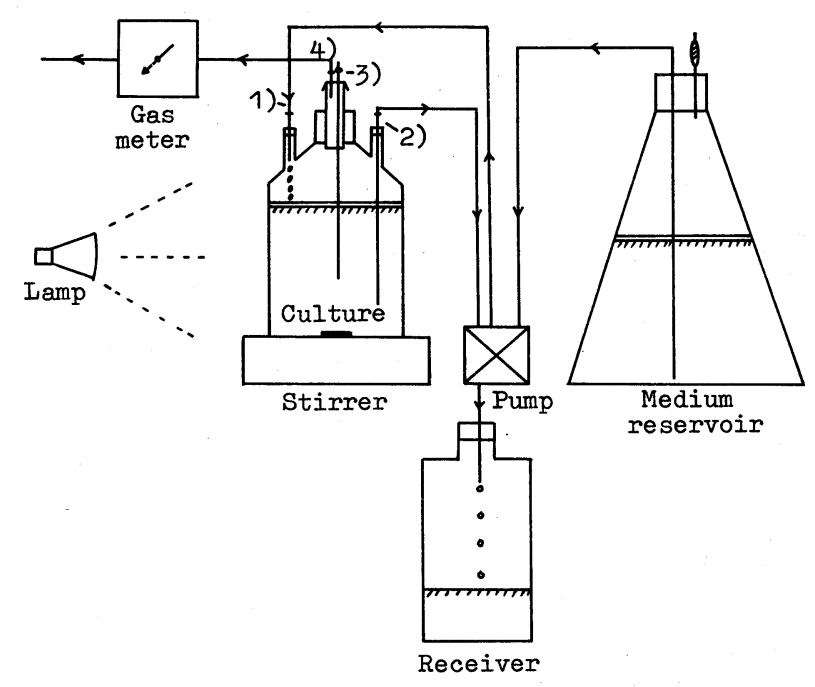

FIG. 1. Apparatus for Continuous Culture for Hydrogen Production.

1) medium inlet; 2) medium outlet; 3) sampling needle; 4) gas outlet. 
previously. ${ }^{3)}$ The amount of hydrogen produced was expressed as its volume under standard conditions. Bacterial growth was followed by measuring the absorbancy at $660 \mathrm{~nm}\left(A_{660} \mathrm{~nm}\right)$ with a Hitachi photoelectric colorimeter, FPW-4. The concenrations of L-glutamate in the media were determined enzymatically with L-glutamate dehydrogenase, ${ }^{5)}$ and DL-lactate was measured colorimetrically by the method of Barker and Summerson. ${ }^{6)}$

The culture under uninterrupted illumination was started using medium A. After incubation for $48 \mathrm{hr}$, fresh medium (A) was supplied at the dilution rate of $0.0411 \mathrm{hr}^{-1}$. As shown in Fig. 2, the steady state of the culture was maintained at $A_{660} \mathrm{~nm}=$ approximately 1.0 , this value corresponded to $0.6 \mathrm{mg}$ cell dry weight $/ \mathrm{ml}$ of the culture. The concentration of lactate in the culture medium ranged between 3.8 and $6.2 \mathrm{~mm}$, which corresponded to 84.8 to $75.2 \%$ consumption of the supplied lactate, respectively. Almost no L-glutamate was detected in the culture medium. The $\mathrm{pH}$ of the medium almost remained completely constant at 7.2 to 7.3 throughout the experiment. Hydrogen was produced at the rate of 70 to $86 \mathrm{ml} / \mathrm{hr}$ per culture (average, $1,874 \mathrm{ml} /$ day). The composition of the evolved gas was $78 \%$ hydrogen and $22 \%$ carbon dioxide on average throughout the culture period. The average rate of hydrogen production was $125.8 \mathrm{ml} / \mathrm{hr} / \mathrm{g}$ dry cells and the average conversion efficiency of consumed lactate to hydrogen was $68.8 \%$ of the theoretical

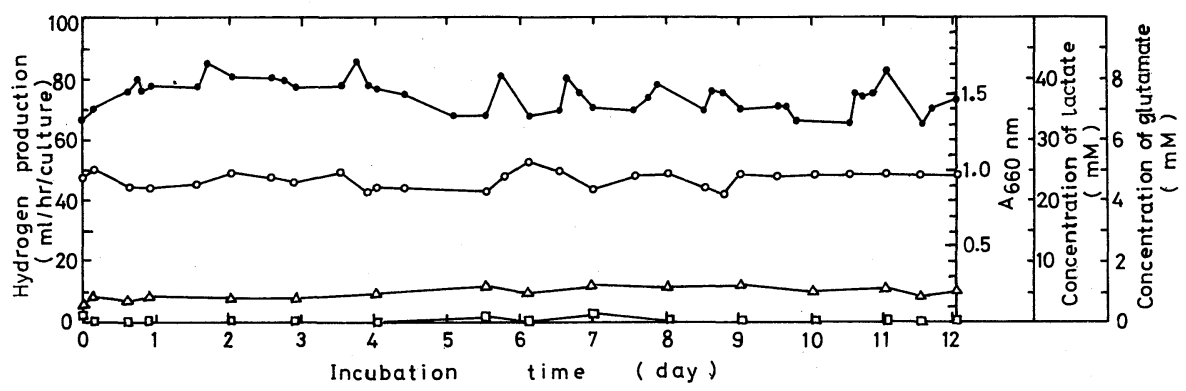

FIG. 2. Hydrogen Production by a Continuous Culture without Interruption of Illumination.

Rate of hydrogen production $(\bigcirc)$, cell density $(\bigcirc)$, concentrations of lactate $(\triangle)$, and L-glutamate $(\square)$.

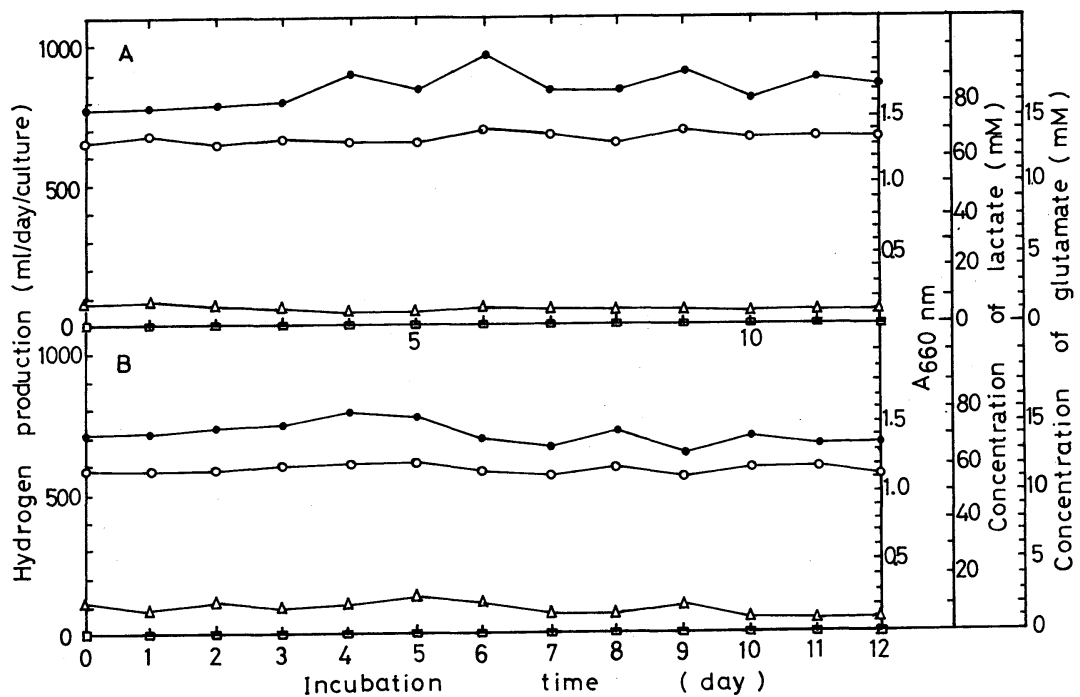

FIG. 3. Hydrogen Production by a Continuous Culture under Illumination for $12 \mathrm{hr}$ Intervals.

Cultures with and without stirring were started using medium B. After incubation for $96 \mathrm{hr}$, fresh medium (B) was supplied to both cultures at the dilution rate of $0.015 \mathrm{hr}^{-1}$. The hydrogen production results in the dark periods are omitted from the time courses in both cases.

A: With stirring. Stirring of the culture was stopped during the dark periods.

B: Without stirring. The symbols are the same as those in Fig. 2. 
value, that is, $6 \mathrm{~mol}$ of hydrogen were produced per mol of lactate.

Zürrer and Bachofen ${ }^{7)}$ reported that on continuous culture of Rhodospirillum rubrum under laboratory conditions 1,250 to $1,550 \mathrm{ml}$ of hydrogen was produced per day per liter culture medium at the rate of $20 \mathrm{ml} / \mathrm{hr} / \mathrm{g}$ dry cells (the culture contained 3.0 to $3.5 \mathrm{mg}$ dry cells $/ \mathrm{ml}$ ), with a yield of $65 \%$. We obtained more hydrogen per liter and a much higher specific activity. This may have been due to the use of a more active strain and of stronger illumination in the present study.

In order to examine the effect of the illumination interval on hydrogen production, we conducted cultures of strain B6 under periodic illumination for $12 \mathrm{hr}$ intervals with and without stirring. In the dark period, feeding of the culture medium was stopped for both culture conditions.

Using medium B, the steady state of both cultures was obtained with a dilution rate of $0.015 \mathrm{hr}^{-1}$, and at $A_{660} \mathrm{~nm}=$ around 1.3 in the stirred culture and at $A_{660} \mathrm{~nm}=$ around 1.15 in the culture without stirring. As shown in Fig. 3, about 840 and $700 \mathrm{ml}$ of hydrogen per liter per day was produced with conversion efficiencies of 73.9 and $61.6 \%$ in the cultures with and without stirring, respectively. There was a short lag time betore hydrogen production in both cultures at the beginning of illumination.

Since hydrogen production continued at almost the same rate under repeated periodic illumination, even without stirring, the results indicate that this bacterium is suitable for daytime continuous outdoor culture for hydrogen production.

Acknowledgment. This investigation was supported by Grants-in-Aid for Developmental Scientific Research (to H. T., Nos. 586025 and 57860015) from the Ministry of Education, Science and Culture of Japan.

\section{REFERENCES}

1) J. S. Kim, K. Ito, K. Izaki and H. Takahashi, Agric. Biol. Chem., 51, 1173 (1987).

2) K. Watanabe, J. S. Kim, K. Ito, L. Buranakarl, T. Kampee and H. Takahashi, Agric. Biol. Chem., 45, 217 (1981).

3) J. S. Kim, K. Ito and H. Takahashi, Agric. Biol. Chem., 46, 937 (1982).

4) J. S. Kim, K. Ito and H. Takahashi, J. Ferment. Technol., 59, 185 (1981)

5) E. Bernt and H. U. Bergmeyer, "Methods of Enzymatic Analysis, "Vol. 4, ed. by H. U. Bergmeyer, Academic Press Inc., New York, 1974, pp. $1704 \sim 1708$.

6) S. B. Barker and W. H. Summerson, J. Biol. Chem., 138, 535 (1941).

7) H. Zürrer and R. Bachofen, Appl. Environ. Microbiol., 37, 789 (1979). 